\title{
Leader localization in multi-agent systems subject to failure: A graph-theoretic approach ${ }^{\star}$
}

\author{
Saeid Jafari ${ }^{\text {a }}$, Amir Ajorlou ${ }^{\text {a }}$, Amir G. Aghdam ${ }^{\text {a }}$ \\ ${ }^{a}$ Department of Electrical and Computer Engineering, Concordia University, 1455 De Maisonneuve Blvd. W., Montréal, \\ Québec, H3G 1M8, Canada
}

\begin{abstract}
In this paper, structural controllability of a leader-follower multi-agent system with multiple leaders is studied. A graphical condition for structural controllability based on the information flow graph of the system is provided. The notions of $p$ link and $q$-agent controllability in a multi-leader setting are then introduced, which provide quantitative measures for the controllability of a system which is subject to failure in the agents and communication links. The problem of leader localization is introduced, which is concerned with finding the minimum number of agents whose selection as leaders results in a $p$-link or $q$-agent controllable network. Polynomial-time algorithms are subsequently presented to solve the problem for both cases of undirected and directed information flow graphs.
\end{abstract}

Key words: Multi-agent systems; controllability; graph theory.

\section{Introduction}

There has been a surge of interest in the use of multiagent systems in a wide variety of engineering applications over the past several years. Important features of multi-agent systems and their superiority to traditional monolithic systems in terms of reliability, flexibility, and adaptability to unknown dynamic environments have been extensively investigated $[4,14,35]$. In particular, control and coordination of this type of system have received a great deal of interest in recent years $[1,2,10,16,24]$. Cooperative control of multi-agent systems has a broad range of applications including formation flying of multiple unmanned aerial vehicles. Due to the importance of information sharing in the coordination of agents, the information flow structure of the system must be taken into consideration in any control design algorithm $[17,21,23]$.

Graph-theoretic techniques, on the other hand, are popular and effective tools for the analysis of multi-agent

\footnotetext{
* This work has been supported in part by the Natural Sciences and Engineering Research Council of Canada (NSERC) under Grant STPGP-364892-08, and in part by Motion Metrics International Corp.

Email addresses: s_jafar@ece.concordia.ca (Saeid Jafari), a_ajor@ece.concordia.ca (Amir Ajorlou), aghdam@ece.concordia.ca (Amir G. Aghdam).
}

systems. Such tools are often employed to analyze a number of related problems such as consensus, rendezvous, flocking, containment and leader-follower formation control, to name only a few $[3,5,6,26,30]$. The controllability problem in leader-follower multi-agent systems was first introduced in [33], where the classical notion of controllability was studied for a leader-based multi-agent system. Necessary and sufficient conditions were subsequently derived for the controllability of the system in terms of the eigenvalues and eigenvectors of a submatrix of the graph's Laplacian. It was also substantiated in [33] that increasing the size of the information flow graph would not necessarily improve the controllability of the system. In [29], it was shown that a leader-symmetric interconnection network is uncontrollable. Network equitable partitions were introduced in [13] to present a new necessary condition for the controllability of a multi-agent system. Using this notion, the controllability characterization methods were extended to the multiple-leader case [28]. More recently, the notion of relaxed equitable partitions was introduced in [22] to provide a graph-theoretic interpretation for the controllability subspace when the network is not completely controllable. The controllability of a single-leader multiagent system under fixed and switching topologies for both continuous-time and discrete-time cases was studied in $[18,19]$, where it was shown that the controllability of the overall system does not require that the network be controllable for a fixed topology. In [36], the structural 
controllability of multi-agent systems was studied using weighted communication links. The case of an information flow graph with switching topology was investigated in [20]. Graphical characterization of the structural controllability for high-order multi-agent systems was given in [27]. The effect of leader selection and weight assignment on controllability was discussed in [34].

In [12], the structural controllability of a single-leader multi-agent system was studied. Then, the notions of $p$ link and $q$-agent controllability were introduced as quantitative measures for the structural controllability of a system subject to failure in communication links and agents. Topology-based necessary and sufficient conditions were also given in [12] for controllability preservation under such failures. Polynomial-time algorithms were subsequently developed to find the maximum number of such failures for which the system remains structurally controllable. The results of [12] are very useful in the controllability analysis of a single-leader configuration; however, many real-world multi-agent system applications require more than one leader to ensure controllability (the interested reader is referred to $[11,15,25,32]$ for some relevant problems).

In this paper, the structural controllability of a leaderfollower multi-agent system with multiple leaders is investigated. A necessary and sufficient condition is provided, which extends the results of [12] to the multipleleader case. A necessary and sufficient condition is also provided for the controllability of the system when some of the communication links between the agents are disabled. This gives a quantitative measure for the reliability of the multi-agent system with respect to its communication links, and can analogously be extended to the case of the agent loss. The problem of leader localization is subsequently introduced, where it is desired to determine the minimum number of leaders (to be selected from agents) for which the system maintains a certain degree of controllability. This helps the designer to improve the reliability and efficiency of a multi-agent system by properly assigning leaders. The present work has several advantages over the existing literature. First of all, network controllability in the case of link/agent failure for a multiple leader setting has not been studied in prior literature. Furthermore, no optimal procedure for leader selection has been reported in the literature so far. In addition, the present work deals with the more general case of directed graphs, as opposed to undirected graphs.

This paper is organized as follows: Section 2 provides some preliminaries from graph theory, and introduces the notation used throughout the paper. The problem is defined and formulated in Section 3. Sections 4 and 5 present the main results of the paper. First, Section 4 extends the definition of structural controllability of singleleader multi-agent systems to the multiple-leader case and provides necessary and sufficient conditions for that.
The results are then used in Section 5 to find a minimum number of agents, which if selected as leaders, the overall system becomes structurally controllable. Finally, the concluding remarks are summarized in Section 6 .

\section{Preliminaries and notation}

Throughout this paper, the set of integers $\{1,2, \ldots, k\}$ is denoted by $\mathbb{N}_{k}$. The difference of the sets $X$ and $Y$, that is the set of those elements of $X$ which do not belong to $Y$ is denoted by $X \backslash Y$. The size of a set $X$ is the number of its elements, and is represented by $|X|$. The $i$ th member of an ordered set $X$ is denoted by $X(i)$. Two sets $X$ and $Y$ are intersecting if the sets $X \backslash Y, Y \backslash X$, and $X \cap Y$ are all nonempty. A directed graph or digraph $\mathcal{G}$ is defined by a set of vertices $V=\{1, \ldots, n\}$ and a set of edges $E \subseteq V \times V$, and is represented by $\mathcal{G}=(V, E)$. A directed arc from vertex $i$ to vertex $j$ is denoted by the edge $e_{i j}:=(i, j) \in E$. In such an ordered pair, the first vertex $i$ is called a tail and the second vertex $j$ is called a head. A self-loop $e_{i i}=(i, i)$ is an edge connecting vertex $i$ to itself. Two edges are anti-parallel if one's head/tail is the other's tail/head. The set of all neighbors of vertex $i$ is defined as $N_{i}:=\left\{j \mid e_{j i} \in E\right\}$. The sizes of the vertex set and the edge set of a graph are called the order and size of the graph, respectively. A sequence of edges $\left(i_{1}, i_{2}\right),\left(i_{2}, i_{3}\right), \ldots,\left(i_{k-1}, i_{k}\right)$ is referred to as an $i_{1} i_{k}$-path $\left(i_{j} \in V, j \in \mathbb{N}_{k}\right)$, and the parent function of this path is defined as $\zeta\left(i_{j}\right)=i_{j-1}$, for any $j \in \mathbb{N}_{k} \backslash\{1\}$. The vertex $i_{1}$ in the above path is called the origin or root, and the vertex $i_{k}$ is called the end of this path. Two paths are called disjoint if they consist of disjoint sets of vertices. An $R$-rooted path is a path whose origin is in the set $R \subset V$; the set $R$ associated with such a path is called the root set. A vertex $i$ is called reachable from the set $R$ if there exists an $R$-rooted path whose end is the vertex $i$. A group of mutually disjoint $R$-rooted paths is called an $R$-rooted path family. A closed path consisting of distinct vertices is called a cycle. A set of disjoint cycles is called a cycle family. The length of a path or a cycle is the number of its edges (excluding self-loop edges). The set of all edges of $\mathcal{G}$ entering $X \subseteq V$ is denoted by $\partial_{\mathcal{G}}^{-}(X)$, and is called the incut of $X$. The set of all edges of $\mathcal{G}$ leaving $X$, on the other hand, is denoted by $\partial_{\mathcal{G}}^{+}(X)$, and is referred to as the outcut of $X$. The size of the incut and outcut associated with $X$ are denoted by $d_{\mathcal{G}}^{-}(X)$ and $d_{\mathcal{G}}^{+}(X)$, and are called the indegree and outdegree of $X$, respectively. For two disjoint sets $X, Y \subset V$, let $\partial_{\mathcal{G}_{Y}}^{-}(X) \subseteq \partial_{\mathcal{G}}^{-}(X)$ be the set of all edges of $\mathcal{G}$ whose tails lie in $Y$ and whose heads lie in $X$; denote the size of this set with $d_{\mathcal{G}_{Y}}^{-}(X)$.

An undirected graph is a graph whose edges are all undirected (represented by plain lines). Throughout this paper, an undirected graph (and its edge set) will be distinguished by a bar over the symbol. A chord of a cycle in an undirected graph $\overline{\mathcal{G}}$ is an edge between two vertices of the cycle that is not an edge of the cycle. The 
graph $\overline{\mathcal{G}}$ is called chordal if any cycle of length greater than three has at least one chord. A clique of $\overline{\mathcal{G}}$ is a set of mutually adjacent vertices. A clique cover is a family of cliques that includes every vertex of the graph. Finding the minimum number of cliques which cover all vertices of a graph is known as the minimum clique cover problem. Given an undirected graph $\overline{\mathcal{G}}=(V, \bar{E})$, its directed counterpart will be represented by $\overrightarrow{\mathcal{G}}$, which is a digraph obtained by replacing every edge of $\overline{\mathcal{G}}$ with a pair of antiparallel directed edges.

In a graph representing a leader-follower multi-agent system, where each vertex corresponds to an agent, the set of vertices corresponding to the leading agents is specified as the root set $R$.

A matrix is called structured if its entries are either fixed zeros or independent free parameters. Let $A \in \mathbb{R}^{n \times n}$ and $B \in \mathbb{R}^{n \times m}$ be two structured matrices. A linear time-invariant (LTI) system whose state-space equation in the standard form is described by the structured pair $(A, B)$ is called a structured system. The $m$-input, $n$ dimensional structured system $\mathbf{S}$ defined by the pair $(A, B)$ can be represented by a digraph $\mathcal{G}_{\mathbf{s}}$ with $n+m$ vertices, where the $i j$ th entry of matrix $[A \mid B]$ is associated with a directed edge from vertex $j$ to vertex $i$, if this entry is nonzero. A structured system $(A, B)$ is said to be structurally controllable if its free parameters can be set to some particular values for which the system is controllable.

\section{Problem statement}

Consider a team of single integrator agents given by

$$
\dot{x}_{i}(t)=u_{i}(t), \quad i \in \mathbb{N}_{n}
$$

where $x_{i}(t)$ and $u_{i}(t)$ are, respectively, the state and control input of agent $i$. The interaction structure between the agents is specified by an information flow graph $\mathcal{G}=(V, E)$, which is known a priori, and is assumed to be static and directed. Let the order of the digraph $\mathcal{G}$ be $n$. There is a directed edge from vertex $j$ to vertex $i$, if agent $j$ transmits its state to agent $i$ (note that each vertex corresponds to an agent, as pointed out before). Assume that some of the agents, say the last $m$ agents, act as leaders and are influenced by external control inputs denoted by $u_{i}(t)=u_{\text {ext }}^{i}(t), i \in \mathbb{N}_{n} \backslash \mathbb{N}_{n-m}$, enabling them to move without any constraint. The rest of the agents, called followers, are governed by the following control law

$$
u_{i}(t)=\sum_{j \in N_{i} \cup\{i\}} \alpha_{i j} x_{j}(t), \quad i \in \mathbb{N}_{n-m}
$$

where the coefficients $\alpha_{i j} \in \mathbb{R}$ are fixed. The state of each agent is defined to be its absolute position with respect to (w.r.t) an inertial reference frame, which can often be measured with sufficient accuracy by using GPS-based systems. Throughout the paper, it is assumed that the agent dynamics is decoupled along each axis, allowing for one-dimensional state representation for each agent.

Definition 1 [12] The information flow graph $\mathcal{G}$ is called controllable if one can choose $\alpha_{i j}$ 's in (2) in such a way that by moving the leaders properly, the followers can take any desired configuration.

Note that in the above definition it is implicitly assumed that each agent is able to detect the indices of its neighbors, so that it can properly multiply the position of each neighbor by the coefficient associated with it in (2). Under the given control law, the dynamics of the followers can be described as

$$
\dot{x}(t)=A x(t)+B u(t)
$$

where $x(t)=\left[\begin{array}{lll}x_{1}(t) \ldots & x_{n-m}(t)\end{array}\right]^{T} \in \mathbb{R}^{n-m}, u(t)=$ $\left[x_{n-m+1}(t) \ldots x_{n}(t)\right]^{T} \in \mathbb{R}^{m}$. Furthermore, the matrices $A=\left[a_{i j}\right]$ and $B=\left[b_{i j}\right]$ are structured matrices [31] of proper dimensions. The state equation (3) describes a structured system whose controllability is equivalent to the controllability of the underlying information flow graph $\mathcal{G}$. It is to be noted that although only the dynamics of the followers are considered in (3), the controllability of the corresponding information flow graph implies that all agents (including the leaders) can reach any desired position by a proper choice of the external input for the leaders.

The above setting includes the applications where the agents can exchange information through communication channels (whose topology is represented by an information flow graph). These communication links are subject to failure in practice. Hence, for a reliable design, one should take into account the controllability of the system in the case of failure of a limited number of communication links. Under some conditions, this can be guaranteed by a proper selection of leaders, which is thoroughly investigated in this work. More precisely, it is desired in this paper to determine the minimum number of leaders required to achieve structural controllability. This problem, which is hereafter referred to as the leader localization problem, is addressed in the general case of a multi-agent system subject to failure in some communication links or loss of some agents.

\section{Structural controllability of multi-agent sys- tems}

In [12], the controllability of the information flow graph of a single-leader multi-agent system is studied. This section aims to extend the results of [12] to a multiple leader setting, that is when more than one agent can 
act as leaders. Consider a structured system with the state-space representation of the form (3). The following theorem is borrowed from [7], and provides necessary and sufficient conditions for structural controllability of the system in terms of its digraph.

Theorem 1 [7] A structured system $\mathbf{S}$ of the form (3) with the digraph $\mathcal{G}_{\mathbf{s}}$ is structurally controllable if and only if both of the following conditions hold:

(i) every vertex in $\mathcal{G}_{\mathbf{s}}$ is the end vertex of an $R$-rooted path, and

(ii) there exists a disjoint union of an $R$-rooted path family and a cycle family that covers all vertices,

where $R \subset V_{\mathbf{s}}$ is the set of vertices corresponding to the columns of the matrix $B$.

The following theorem provides a necessary and sufficient condition for the structural controllability of an information flow graph.

Theorem 2 The information flow graph $\mathcal{G}$ is controllable if and only if every vertex in $V \backslash R$ is reachable from the root set $R$.

Proof: The reachability of every vertex of the set $V \backslash R$ from the root set $R$ is equivalent to every member of $V \backslash R$ being the end vertex of an $R$-rooted path in $\mathcal{G}_{\mathbf{s}}$, which is condition $(i)$ of Theorem 1 . The vertices in $R$ can be considered as $R$-rooted paths of length zero, and the selfloops in the vertices of $V \backslash R$ constitute a cycle family whose union with these zero-length $R$-rooted paths span the vertex set $V=V_{\mathbf{s}}$. This implies that condition $(i i)$ of Theorem 1 is always satisfied for the information flow graph of a system with control law of form (2). The proof follows now from Theorem 1.

Definition 2 [12] The information flow graph $\mathcal{G}$ is said to be p-link controllable if $p$ is the largest number for which the controllability of $\mathcal{G}$ is preserved after removing any group of at most $p-1$ edges.

In a $p$-link controllable digraph, $p$ is the minimum number of edges whose removal makes the digraph uncontrollable. For a digraph $\mathcal{G}$ with the root set $R$, this number will hereafter be denoted by $l c(\mathcal{G} ; R)$, and will be referred to as the link-controllability degree of $\mathcal{G}$. Define $l c(\mathcal{G} ; \emptyset)=0$ and $l c(\mathcal{G} ; V)=\infty$, and let $l c(\mathcal{G}, x ; R)$ be the minimum number of edges whose removal makes the vertex $x \in V \backslash R$ unreachable from the root set $R$. Clearly, $l c(\mathcal{G} ; R)=\min _{x \in V \backslash R} l c(\mathcal{G}, x ; R)$. The set $R$ is called a $p$-link root set if $l c(\mathcal{G} ; R) \geq p$. It is important to note that $l c(\mathcal{G} ; R)$ is, in fact, a quantitative measure for the reliability of the multi-agent system w.r.t. communication failure. Note also that the concept of $p$-link controllability is closely related to the notion of rooted-edgeconnectivity in graph theory [8]. The following theorem gives a necessary and sufficient condition for the $p$-link controllability based on the information flow graph $\mathcal{G}$.

Theorem 3 The information flow graph $\mathcal{G}=(V, E)$ with the root set $R$ is p-link controllable if and only if

$$
\min _{R \subseteq X \subset V} d_{\mathcal{G}}^{+}(X)=p
$$

Proof: It is clear from the definition of outcut that removing the set $\partial_{\mathcal{G}}^{+}(X)$ from the edge set $E$ for every $X \subset V$ containing $R$ (i.e. $R \subseteq X$ ) makes the set $V \backslash X$ unreachable from $R$. On the other hand, suppose that $F$ is the minimal set of edges whose removal makes at least one of the vertices unreachable from $R$, and let $X_{F}$ be the set of reachable vertices from $R$ after the removal of those edges. The proof follows now on noting that $F$ includes all members of the outcut of $X_{F}$, i.e., $\partial_{\mathcal{G}}^{+}\left(X_{F}\right) \subseteq F$.

One can use Theorem 3 to find the value of $l c(\mathcal{G} ; R)$ in any digraph $\mathcal{G}$. However, calculating the outdegree of all possible subsets of the vertex set $V$ takes exponential time, and hence is intractable for high-order digraphs. Therefore, it is desired to develop a polynomial-time algorithm to obtain this value for any digraph.

To find $l c(\mathcal{G}, x ; R)$, let a new digraph $\mathcal{G}^{\prime}=\left(V^{\prime}, E^{\prime}\right)$ be constructed from $\mathcal{G}$ by extending the sets $V$ and $E$ as follows: Consider a new vertex $r$, and define $V^{\prime}=V \cup\{r\}$ and $E^{\prime}=E \cup\{(r, i), \forall i \in R\}$. The digraph $\mathcal{G}^{\prime}$ will be referred to as the expanded digraph of $\mathcal{G}$ w.r.t. $R$. As an illustrative example, Fig. 1(a) shows a digraph $\mathcal{G}$ with the root set $R=\{4,5,6\}$, and Fig. 1(b) demonstrates how the $\operatorname{digraph} \mathcal{G}^{\prime}$ is constructed from $\mathcal{G}$.

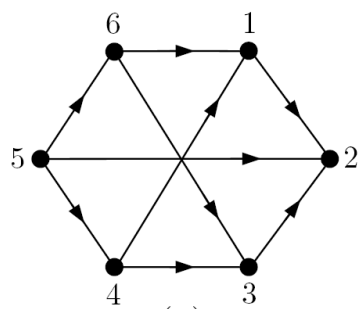

(a)

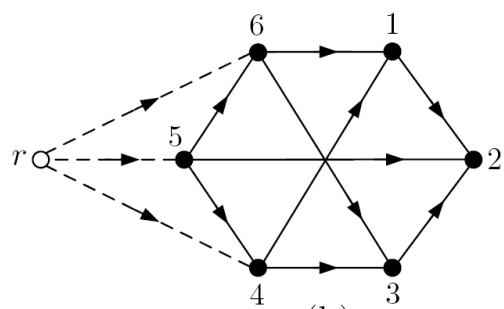

(b)

Fig. 1. (a) An information flow graph $\mathcal{G}$, and (b) the corresponding expanded digraph $\mathcal{G}^{\prime}$ w.r.t. $R=\{4,5,6\}$. 
Consider the expanded digraph $\mathcal{G}^{\prime}=\left(V^{\prime}, E^{\prime}\right)$ corresponding to a given digraph $\mathcal{G}$ and the root set $R$, and let $x \in V^{\prime} \backslash\{R \cup\{r\}\}$ be a specified vertex of $\mathcal{G}^{\prime}$. Construct a new digraph $\mathcal{G}_{\text {new }}^{\prime}$ by reversing the direction of all edges of any $r x$-path, except for those edges which belong to $\{r\} \times R$, if any. It can be shown that by reversing the directions of such edges of an $r x$-path, $l c\left(\mathcal{G}^{\prime}, x ; r\right)$ will be reduced by one [12]. Repeat the same procedure for $\mathcal{G}_{\text {new }}^{\prime}$ and continue until a digraph $\mathcal{G}_{\text {final }}^{\prime}$ is obtained in which $x$ is unreachable from the root $r$. Denote with $X_{r, \mathcal{G}^{\prime}}$ the set of all reachable vertices from $r$ in $\mathcal{G}_{\text {final }}^{\prime}$ (note that $\left.X_{r, \mathcal{G}^{\prime}} \subset V^{\prime}\right)$.

Theorem 4 The outcut of $X_{r, \mathcal{G}^{\prime}}$ in $\mathcal{G}^{\prime}$ is a minimal set whose removal makes the vertex $x \in V^{\prime} \backslash\{R \cup\{r\}\}$ unreachable from $R$; in particular, $d_{\mathcal{G}^{\prime}}^{+}\left(X_{r, \mathcal{G}^{\prime}}\right)=l c(\mathcal{G}, x ; R)$.

Proof: Each time the directions of the edges of an $r x$ path are reversed, the outdegree of $X_{r, \mathcal{G}^{\prime}}$ decreases by one [12]. The proof follows now from the fact that for the final digraph $\mathcal{G}_{\text {final }}, \partial_{\mathcal{G}_{\text {final }}}^{+}\left(X_{r, \mathcal{G}^{\prime}}\right)=\emptyset$.

One can use Theorem 4 to develop a polynomial-time procedure for finding the value of $l c(\mathcal{G}, x ; R)$. The following algorithm is presented for this purpose.

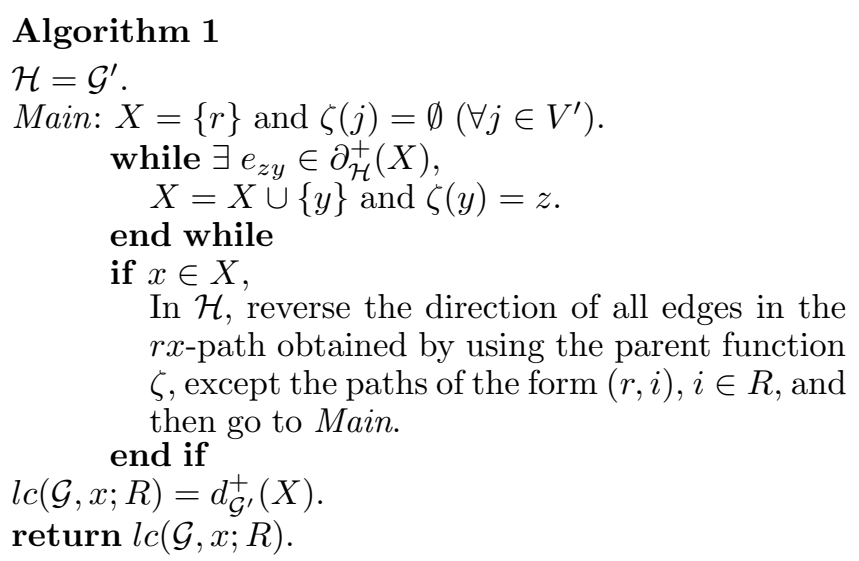

As an example, by applying the above algorithm to the digraph shown in Fig. 1 , one will obtain $l c(\mathcal{G}, 1 ; R)=$ $2, l c(\mathcal{G}, 2 ; R)=3$, and $l c(\mathcal{G}, 3 ; R)=2$; hence, for this digraph $l c(\mathcal{G} ; R)=2$.

Remark 1 Analogously to the problem of p-link controllability, one can define the problem of q-agent controllability, which is concerned with the controllability preservation after the failure of at most $q-1$ agents [12]. This problem can be converted to the problem of q-link controllability by using the node-duplication technique as discussed in [12].

\section{Leader localization}

Leader localization in a multi-agent system deals with the problem of finding a minimum number of agents, which if selected as leaders, the overall system becomes structurally controllable. Given a positive integer $p(p<$ $n$ ), it is desired in the sequel to find a vertex set $R \subseteq$ $V$ of the smallest size such that the information flow graph $\mathcal{G}$ is at least $p$-link controllable. To this end, some important ideas are borrowed from $[32,11]$.

Definition 3 Given a digraph $\mathcal{G}=(V, E)$, a set $X \subset V$ is called $p$-deficient if $d_{\mathcal{G}}^{-}(X)<p$. A p-deficient set is minimal if none of its proper subsets is p-deficient. As an example, Fig. 2 shows a digraph with two minimal 2deficient sets $X_{1}=\{1,2,3\}$ and $X_{2}=\{4\}$.

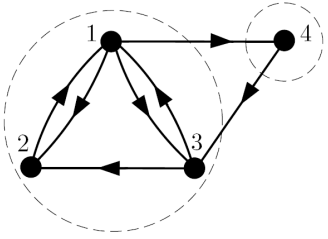

Fig. 2. A digraph with two minimal 2-deficient sets.

Theorem 5 Given a digraph $\mathcal{G}=(V, E)$, a set $R \subseteq V$ is a p-link root set if and only if any $p$-deficient set $\bar{X}$ in $\mathcal{G}$ intersects $R$.

Proof: Assume $X$ is a $p$-deficient set disjoint from $R$. Since $d_{\mathcal{G}}^{-}(X)<p$, hence for every vertex $x \in X$, $l c(\mathcal{G}, x ; R)<p$. This contradicts the initial assumption that $R$ is a $p$-link root set. Consider now a set $R(R \subseteq V)$ for which $l c(\mathcal{G} ; R)<p$, and assume $R$ intersects any $p$-deficient set of $\mathcal{G}$. According to Theorem 3, there exists a set $X \subset V$ containing $R$, such that $d_{\mathcal{G}}^{+}(X)<p$, or equivalently $d_{\mathcal{G}}^{-}(V \backslash X)<p$. This means that $V \backslash X$ is a $p$-deficient set disjoint from $R$, which contradicts the assumption that $R$ intersects any $p$-deficient set of $\mathcal{G}$. This contradiction completes the proof.

One can deduce from Theorem 5 that a set $R$ is a $p$ link root set if and only if any minimal $p$-deficient set intersects $R$.

Although undirected graphs can be viewed as a special case of digraphs, because of certain properties they have, one can develop a simpler procedure to solve the leader localization problem for this type of graph. Therefore, the two cases of directed and undirected information flow graphs are considered separately in the next two subsections.

\subsection{Undirected information flow graphs}

This section investigates the leader localization problem in a network with bidirectional communication links, represented by the undirected information flow graph $\overline{\mathcal{G}}=(V, \bar{E})$. To this end, certain properties of undirected graphs (which do not hold for directed graphs, in general) will be discussed in the sequel. 
Let $\overrightarrow{\mathcal{G}}=(V, \vec{E})$ represent the directed counterpart of the undirected graph $\overline{\mathcal{G}}$. It is straightforward to show that $l c(\overline{\mathcal{G}}, x ; R)=l c(\overrightarrow{\mathcal{G}}, x ; R)$, for any $R \subset V$ and $x \in V \backslash R$.

Lemma 1 Let $X$ and $Y$ be two intersecting subsets of $V$, and define $Z=V \backslash\{X \cup Y\}$. Then

$$
\begin{aligned}
d_{\overrightarrow{\mathcal{G}}}^{-}(X)+d_{\overrightarrow{\mathcal{G}}}^{-}(Y)=d_{\overrightarrow{\mathcal{G}}}^{-}(X \backslash Y) & +d_{\overrightarrow{\mathcal{G}}}^{-}(Y \backslash X) \\
& +2 d_{\overrightarrow{\mathcal{G}}_{Z}}^{-}(X \cap Y)
\end{aligned}
$$

Proof: It is straightforward to show that

$$
\begin{aligned}
d_{\overrightarrow{\mathcal{G}}}^{-}(X)= & d_{\overrightarrow{\mathcal{G}}_{Z}}^{-}(X \backslash Y)+d_{\overrightarrow{\mathcal{G}}_{Y \backslash X}}^{-}(X \backslash Y) \\
& +d_{\overrightarrow{\mathcal{G}}_{Z}}^{-}(X \cap Y)+d_{\overrightarrow{\mathcal{G}}_{Y \backslash X}}^{-}(X \cap Y) \\
d_{\overrightarrow{\mathcal{G}}}^{-}(Y)= & d_{\overrightarrow{\mathcal{G}}_{Z}}^{-}(Y \backslash X)+d_{\overrightarrow{\mathcal{G}}_{X \backslash Y}^{-}}(Y \backslash X) \\
+ & d_{\overrightarrow{\mathcal{G}}_{Z}}^{-}(X \cap Y)+d_{\overrightarrow{\mathcal{G}}_{X \backslash Y}}^{-}(X \cap Y) \\
d_{\overrightarrow{\mathcal{G}}}^{-}(X \backslash Y)= & d_{\overrightarrow{\mathcal{G}}_{Z}}^{-}(X \backslash Y)+d_{\overrightarrow{\mathcal{G}}_{Y \backslash X}}^{-}(X \backslash Y) \\
& +d_{\overrightarrow{\mathcal{G}}_{X \cap Y}}^{-}(X \backslash Y) \\
d_{\overrightarrow{\mathcal{G}}}^{-}(Y \backslash X)= & d_{\overrightarrow{\mathcal{G}}_{Z}}^{-}(Y \backslash X)+d_{\overrightarrow{\mathcal{G}}_{X \backslash Y}}^{-}(Y \backslash X) \\
& +d_{\overrightarrow{\mathcal{G}}_{X \cap Y}}^{-}(Y \backslash X)
\end{aligned}
$$

It can also be easily shown that

$$
\begin{aligned}
& d_{\overrightarrow{\mathcal{G}}_{Y \backslash X}}^{-}(X \cap Y)=d_{\overrightarrow{\mathcal{G}}_{X \cap Y}}^{-}(Y \backslash X) \\
& d_{\overrightarrow{\mathcal{G}}_{X \backslash Y}}^{-}(X \cap Y)=d_{\overrightarrow{\mathcal{G}}_{X \cap Y}}^{-}(X \backslash Y)
\end{aligned}
$$

Now, the proof follows directly from (4), (5) and (6).

Theorem 6 Given a graph $\overline{\mathcal{G}}=(V, \bar{E})$, all minimal $p$ deficient sets of its directed counterpart $\overrightarrow{\mathcal{G}}$ are pairwise disjoint.

Proof: Let $X_{1}, X_{2} \subset V\left(X_{1} \neq X_{2}\right)$ be two minimal $p$ deficient sets, and assume $X_{1} \cap X_{2} \neq \emptyset$. It follows from the definition of a minimal $p$-deficient set that $X_{1}$ and $X_{2}$ are intersecting. On the other hand, since $X_{1} \backslash X_{2} \subset X_{1}$ and $X_{2} \backslash X_{1} \subset X_{2}$, hence the sets $X_{1} \backslash X_{2}$ and $X_{2} \backslash X_{1}$ are not $p$-deficient. From Lemma 1

$$
\begin{aligned}
d_{\overrightarrow{\mathcal{G}}}^{-}\left(X_{1}\right)+d_{\overrightarrow{\mathcal{G}}}^{-}\left(X_{2}\right)= & d_{\overrightarrow{\mathcal{G}}}^{-}\left(X_{1} \backslash X_{2}\right)+d_{\overrightarrow{\mathcal{G}}}^{-}\left(X_{2} \backslash X_{1}\right) \\
& +2 d_{\overrightarrow{\mathcal{G}}_{Z}}^{-}\left(X_{1} \cap X_{2}\right)
\end{aligned}
$$

where $Z:=V \backslash\left\{X_{1} \cup X_{2}\right\}$. The facts that $d_{\overrightarrow{\mathcal{G}}}^{-}\left(X_{1}\right)<p$, $d_{\overrightarrow{\mathcal{G}}}^{-}\left(X_{2}\right)<p, d_{\overrightarrow{\mathcal{G}}}^{-}\left(X_{1} \backslash X_{2}\right) \geq p$, and $d_{\overrightarrow{\mathcal{G}}}^{-}\left(X_{2} \backslash X_{1}\right) \geq p$ imply $d_{\overrightarrow{\mathcal{G}}_{Z}}^{-}\left(X_{1} \cap X_{2}\right)<0$. However, this cannot be true because the indegree of a set cannot be negative. This contradiction completes the proof.
Theorems 5 and 6 imply that in an undirected information flow graph $\overline{\mathcal{G}}$, any minimal $p$-link root set contains a vertex from each minimal $p$-deficient set of $\overrightarrow{\mathcal{G}}$. One can use Theorem 6 to find the minimal $p$-link root sets without explicitly identifying the minimal $p$-deficient sets. This is spelled out in the next theorem.

Theorem 7 Given an undirected graph $\overline{\mathcal{G}}$, let $R$ be a $p$ link root set of its directed counterpart $\overrightarrow{\mathcal{G}}$. For any vertex $x \in R$, iflc $(\overrightarrow{\mathcal{G}}, x ; R \backslash\{x\}) \geq p$, then $R \backslash\{x\}$ is a p-link root set as well. Moreover, if $l c(\overrightarrow{\mathcal{G}} ; R \backslash\{x\})<p$, then there exists a minimal $p$-deficient set $X$ whose only common element with $R$ is $x$.

Proof: Case $i)$ Assume that $l c(\overrightarrow{\mathcal{G}}, x ; R \backslash\{x\}) \geq p$. Since $R$ is a $p$-link root set, it intersects any $p$-deficient set. It can be shown that either $R \backslash\{x\}$ intersects any $p$-deficient set too, or there exists a $p$-deficient set $X$ containing $x$, such that $X$ is disjoint from $R \backslash\{x\}$. This implies that $l c(\overrightarrow{\mathcal{G}}, x ; R \backslash\{x\}) \leq d_{\overrightarrow{\mathcal{G}}}^{-}(X)<p$ which is a contradiction. The proof in this case follows from Theorem 5 .

Case ii) Assume now that $l c(\overrightarrow{\mathcal{G}} ; R \backslash\{x\})<p$. This implies that $R \backslash\{x\}$ is not a $p$-link root set. Since $R$ is a $p$-link root set, $x$ should belong to a minimal $p$-deficient set in order for $R \backslash\{x\}$ not to intersect any minimal $p$-deficient set. Clearly, $R \cap X \neq \emptyset$ and $(R \backslash\{x\}) \cap X=\emptyset$, which completes the proof.

Theorem 7 is used next to develop a polynomial-time procedure for finding a minimal $p$-link root set. It is to be noted that the minimal $p$-link root set is not necessarily unique.

\section{Algorithm 2}

$R=V$.

for $i=1$ to $n$,

$$
\begin{aligned}
& \text { if } l c(\overrightarrow{\mathcal{G}}, i ; R \backslash\{i\}) \geq p, \\
& \quad R=R \backslash\{i\} . \\
& \text { end if }
\end{aligned}
$$

end for

\section{return $R$.}

As an example, consider the graph shown in Fig. 3. By applying the above algorithm to this graph, one arrives at $R=\{1,4\}$ as the minimal 3-link root set (which is unique in this case).

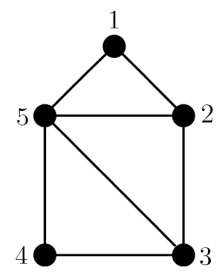

Fig. 3. An undirected information flow graph. 


\subsection{Directed information flow graphs}

The result of Theorem 6 is not valid for a general digraph $\mathcal{G}$, that is, the minimal $p$-deficient sets of $\mathcal{G}$ are not pairwise disjoint in general. Therefore, Algorithm 2 cannot be employed to find a minimal $p$-link root set for an arbitrary digraph. As an example, consider the digraph of order five depicted in Fig. 4. It is desired in this figure to find a minimal 2-link root set. This digraph has two intersecting minimal 2-deficient sets $X_{1}=\{1,2,3\}$ and $X_{2}=\{1,4,5\}$. From Theorem 5 , it can be easily concluded that in this example the minimal 2-link root set is $R=\{1\} \subseteq X_{1} \cap X_{2}$, while Algorithm 2 may give a 2-link root set of size more than one, e.g., $R=\{2,5\}$.

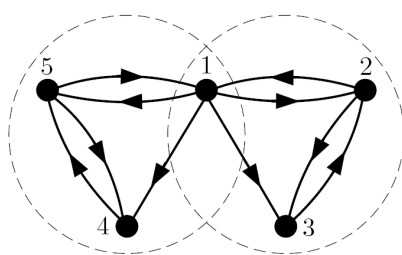

Fig. 4. An information flow graph with two intersecting minimal 2-deficient sets.

According to Theorem 5, a minimal $p$-link root set is a minimal set intersecting all minimal $p$-deficient sets. Therefore, one approach to find a minimal $p$-link root set in a digraph is to construct all minimal $p$-deficient sets, and then to find a minimal set $R$ that intersects all of them.

As an approach for finding all $p$-deficient sets of a digraph $\mathcal{G}=(V, E)$, one can consider all sets $F \subset E$ of size less than $p$, and find all sets $X \subset V$ for which $F=\partial_{\mathcal{G}}^{-}(X)$. Using this approach, one can develop an algorithm with complexity $\mathcal{O}\left(|E|^{p}\right)$ to find the family of all $p$-deficient sets $Q$, where $Q(i)=X_{i}$ denotes the $i$ th $p$-deficient set in $Q$. Although the complexity of such an algorithm grows exponentially with the value of $p$, the set $Q$ can be constructed in polynomial-time in terms of the size of the graph, because $p$ is a fixed (and typically small) number. The following algorithm is derived based on the above-mentioned approach.

\section{Algorithm 3}

$Q=\emptyset$ and $j=1$.

for $k=1$ to $p-1$,

Let $Z$ be the family of all subsets of $E$ of size $k$.

for $i=1$ to $|Z|$, Let $H=\{y \mid(x, y) \in Z(i)\}$ and $T=\{x \mid(x, y) \in$ $Z(i)\}$.

while there exists an edge $(u, v) \in \partial_{\mathcal{G}}^{-}(H)$ and

$(u, v) \notin Z(i)$,

$$
H=H \cup\{u\} \text {. }
$$

end while

if $T \cap H=\emptyset$,

\author{
$Q(j)=H$ \\ $j=j+1$. \\ end if \\ end for \\ end for \\ return $Q$.
}

The members of $Q$ obtained from the above algorithm are not necessarily minimal, i.e., it is possible that $X_{i} \subset$ $X_{j}$, for some distinct $i$ and $j$. To construct the family of all minimal $p$-deficient sets of $\mathcal{G}$, the set $Q$ must be modified by removing any set $X_{j}$ for which there exists a set $X_{i}$ such that $X_{i} \subset X_{j}, i \neq j$. Clearly, this can be performed in polynomial-time. The modified version of $Q$ consisting of only minimal $p$-deficient sets is hereafter denoted by $\hat{Q}$. By applying Algorithm 3 to the digraph shown in Fig. 4 , one arrives at $Q=$ $\{\{1,2,3\},\{1,2,3,5\},\{1,4,5\},\{1,2,4,5\}\}$. Since $X_{1} \subset$ $X_{2}$ and $X_{3} \subset X_{4}$, the family of all minimal $p$-deficient sets will be $\hat{Q}=\left\{X_{1}, X_{3}\right\}=\{\{1,2,3\},\{1,4,5\}\}$.

The following two theorems present some useful properties of minimal $p$-deficient sets of a digraph $\mathcal{G}$, and will be used later to find a minimal $p$-link root set of $\mathcal{G}$. It is to be noted that the main idea of these theorems is borrowed from [11].

Theorem 8 Let $\left\{X_{1}, X_{2}, \ldots, X_{k}\right\} \subseteq \hat{Q}$ be any group of pairwise intersecting members of $\hat{Q}$; then, $\bigcap_{i=1}^{k} X_{i} \neq \emptyset$.

Proof: To prove the theorem by contradiction, assume $W=\left\{X_{1}, \ldots, X_{\ell}\right\} \subseteq \hat{Q}$ is a set with the smallest size $\ell$ for which the statement of the theorem does not hold. This implies that $\bigcap_{X_{i} \in W} X_{i}=\emptyset$, and that for any $W^{\prime} \subset W, \bigcap_{X_{i} \in W^{\prime}} X_{i} \neq \emptyset$. Let $Y_{j}=\bigcap_{X_{i} \in W(i \neq j)} X_{i}$, for $j=1, \ldots, \ell$. It is clear that $Y_{j}$ 's are nonempty pairwise disjoint sets. Also, $\partial_{\mathcal{G}}^{-}\left(Y_{i}\right) \cap \partial_{\mathcal{G}}^{-}\left(Y_{j}\right)=\emptyset$ for any $i \neq j$. This, along with the facts that $\partial_{\mathcal{G}}^{-}\left(Y_{i}\right) \subseteq \bigcup_{j \neq i} \partial_{\mathcal{G}}^{-}\left(X_{j}\right)$ and $X_{i}$ 's are $p$-deficient, implies that $\sum_{i=1}^{\ell} d_{\mathcal{G}}^{-}\left(Y_{i}\right) \leq$ $\sum_{i=1}^{\ell} d_{\mathcal{G}}^{-}\left(X_{i}\right)<p \ell$. This means that $d_{\mathcal{G}}^{-}\left(Y_{i}\right)<p$ for some $i \in \mathbb{N}_{\ell}$; i.e., $Y_{i}$ is also $p$-deficient. This contradicts the minimality assumption for $X_{j}(i \neq j)$, on noting that $Y_{i} \subseteq X_{j}$ for any $i \neq j$. This contradiction completes the proof.

Let $\overline{\mathcal{G}}^{*}=\left(V^{*}, E^{*}\right)$ be an undirected graph of order $|\hat{Q}|$, with a one-to-one correspondence between its vertices and the members of $\hat{Q}$, that is, $X_{i} \in \hat{Q}$ corresponds to vertex $i$ in $\overline{\mathcal{G}}^{*}$. In this graph, two distinct vertices $i, j \in V^{*}$ are adjacent if the corresponding members of $\hat{Q}$ (i.e., $X_{i}$ and $X_{j}$ ) have a nonempty intersection. Then, the problem of finding the minimal $p$-link root set is equivalent to finding the minimum covering by cliques for $\overline{\mathcal{G}}^{*}$. Such a minimal root set can be obtained by considering a common element of the sets corresponding to each clique in this minimum covering (the existence of 
such a common element for each clique is guaranteed by Theorem 8). A special property of $\overline{\mathcal{G}}^{*}$ presented in the next theorem enables one to develop a polynomial-time procedure for finding a minimum covering by cliques for $\overline{\mathcal{G}}^{*}$.

Theorem 9 The graph $\overline{\mathcal{G}}^{*}$ constructed from the family of all minimal p-deficient sets of a digraph $\mathcal{G}$ is chordal.

Proof: Assume $\overline{\mathcal{G}}^{*}$ is not chordal, and has a cycle of length greater than three without any chord. Let the sequence of vertices $1,2, \ldots, \ell$ represent this chordless cycle, and define $Y_{i}=X_{i} \cap X_{i+1}$ for any $i \in \mathbb{N}_{\ell}$. Let also $X_{\ell+1}=X_{1}$, where $X_{i}$ is the minimal $p$-deficient set of $\mathcal{G}$ corresponding to vertex $i \in V^{*}$. It is evident that $Y_{i}^{\prime}$ 's are nonempty pairwise disjoint sets. This implies that $\partial_{\mathcal{G}}^{-}\left(Y_{i}\right) \cap \partial_{\mathcal{G}}^{-}\left(Y_{j}\right)=\emptyset$ for any $i \neq j$. Also, $\partial_{\mathcal{G}}^{-}\left(Y_{i}\right) \subseteq$ $\partial_{\mathcal{G}}^{-}\left(X_{i}\right) \cup \partial_{\mathcal{G}}^{-}\left(X_{i+1}\right)$. The above derivation together with the fact that $X_{i}$ 's are $p$-deficient, yields $\sum_{i=1}^{\ell} d_{\mathcal{G}}^{-}\left(Y_{i}\right) \leq$ $\sum_{i=1}^{\ell} d_{\mathcal{G}}^{-}\left(X_{i}\right)<p \ell$. This implies that $d_{\mathcal{G}}^{-}\left(Y_{i}\right)<p$ for some $i \in \mathbb{N}_{\ell}$, i.e., $Y_{i}$ is $p$-deficient. This contradicts the initial assumption of the minimality of $X_{i}$, as $Y_{i} \subseteq X_{i}$. This contradiction completes the proof.

In [9], a polynomial-time algorithm is proposed to find the minimum covering by cliques for chordal graphs. In order to use this algorithm, it is first required to rename the vertices and properly orient the edges of $\overline{\mathcal{G}}^{*}$ as follows.

\section{Algorithm 4}

$v=\left|V^{*}\right|$.

Mark all vertices of $\overline{\mathcal{G}}^{*}$.

while $\overline{\mathcal{G}}^{*}$ has more than one marked vertex,

if there exists a marked vertex $i$ such that all of its marked neighbors form a clique, then,

Rename $i$ to $v$ and let $\phi(v)=i$.

end if

Unmark $i$ and let $v=v-1$.

\section{end while}

Rename the remaining vertex $j$ to 1 and let $\phi(1)=j$.

for any edge $e_{i j} \in E^{*}$,

Orient $e_{i j}$ from $\min (i, j)$ to $\max (i, j)$.

end for

Let $\overrightarrow{\mathcal{G}}^{*}=\overline{\mathcal{G}}^{*}$.

return $\overrightarrow{\mathcal{G}}^{*}$.

Let $N_{i}^{*}$ be the set of all neighbors of vertex $i$ in $\overrightarrow{\mathcal{G}}^{*}$, and define a sequence of vertices $n_{1}, n_{2}, \ldots, n_{\nu}$ as follows: $n_{1}=\left|V^{*}\right|$ and $n_{k}=\max \left\{i \in V^{*} \mid i<n_{k-1}, i \notin\right.$ $\cup_{j=1}^{k-1} N_{n_{j}}^{*}$. For any $i \in \mathbb{N}_{\nu}$, let $P_{i}=N_{n_{i}}^{*} \cup\left\{n_{i}\right\}$; then, $C_{i}=\bigcup_{j \in P_{i}} \phi(j)$ is a clique and the family $\left\{C_{1}, C_{2}, \ldots, C_{\nu}\right\}$ is a minimum covering by cliques for the graph $\overline{\mathcal{G}}^{*}$.

By retrieving the original labels of the vertices of $\overline{\mathcal{G}}^{*}$ using the function $\phi$, one can construct a minimal $p$-link root set of $\mathcal{G}$ as follows: Let $Y_{i}=\cap_{j \in C_{i}} X_{j}$, for any $i \in \mathbb{N}_{\nu}$. From Theorem $8, Y_{i} \neq \emptyset$, for any $i \in \mathbb{N}_{\nu}$. Therefore, by taking one element from each $Y_{i}$, a minimal $p$-link root set of $\mathcal{G}$ is obtained.

As an example, consider a digraph $\mathcal{G}$ with six minimal $p$-deficient sets $X_{1}, \ldots, X_{6}$, where the graph $\overline{\mathcal{G}}^{*}$ corresponding to its minimal $p$-deficient sets is shown in Fig. 5. Using Algorithm 4, a digraph $\overrightarrow{\mathcal{G}}^{*}$ is obtained

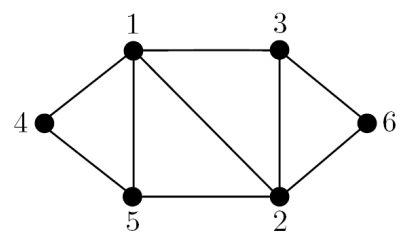

Fig. 5. The graph $\overline{\mathcal{G}}^{*}$ corresponding to all minimal $p$-deficient sets of the digraph $\mathcal{G}$.

from $\overline{\mathcal{G}}^{*}$ by renaming its vertices and orienting the edges. Choose the vertices of $\overline{\mathcal{G}}^{*}$ in the order $(4,6,5,2,1,3)$. Then, $\phi(6)=4, \phi(5)=6, \phi(4)=5, \phi(3)=2, \phi(2)=1$, and $\phi(1)=3$. The digraph $\overrightarrow{\mathcal{G}}^{*}$ shown in Fig. 6 is the outcome of the above procedure.

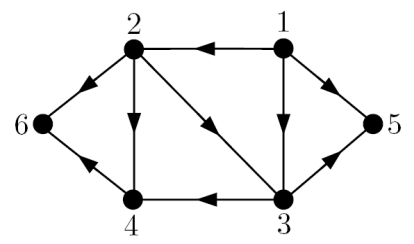

Fig. 6. The digraph $\overrightarrow{\mathcal{G}}^{*}$ obtained from $\overline{\mathcal{G}}^{*}$.

Note in $\overrightarrow{\mathcal{G}}^{*}$ that $n_{1}=6, N_{6}^{*}=\{2,4\}, n_{2}=5$, $N_{5}^{*}=\{1,3\}, P_{1}=\{2,4,6\}$, and $P_{2}=\{1,3,5\}$. Thus, $C_{1}=\{\phi(2), \phi(4), \phi(6)\}=\{1,5,4\}$ and $C_{2}=$ $\{\phi(1), \phi(3), \phi(5)\}=\{3,2,6\}$ are two cliques of $\mathcal{G}^{*}$ and $\{\{1,5,4\},\{3,2,6\}\}$ is a minimum covering by cliques. Now, let $Y_{1}=X_{1} \cap X_{5} \cap X_{4}$ and $Y_{2}=X_{3} \cap X_{2} \cap X_{6}$, and assume $r_{1} \in Y_{1}$ and $r_{2} \in Y_{2}$. Therefore, $R=\left\{r_{1}, r_{2}\right\} \subset V$ is a minimal $p$-link root set for $\mathcal{G}$.

\section{Conclusions}

The structural controllability of a team of single integrator agents is investigated in this work. A leaderfollower formation configuration is considered, where multiple agents can simultaneously act as leaders. Necessary and sufficient conditions for the system to remain structurally controllable in the case of the failure of some of the communication links are derived in terms of the topology of the information flow graph. Then the problem of leader localization is investigated, where it is aimed to find a minimal set of agents that if selected as leaders, the resultant information flow graph 
is $p$-link controllable. This problem is addressed for both undirected and directed interconnection networks, and polynomial-time algorithms are developed for both cases. Analogous results can also be developed for the case of agents loss. More research needs to be done to determine whether or not the results are still valid if the agents use the relative positions of the neighbors instead of the absolute positions. Future research is also planned to investigate the weight assignment for structurally controllable graphs, and to extend the results obtained here to the information flow graphs with randomly switching topologies. Also, this work only considers failure in the inter-agent communication channels. Failure in the communication with GPS in addition to the inter-agent communication links is also an interesting problem that can be considered for future work.

\section{References}

[1] A. G. Aghdam. Robust degree of exponential stability in polynomially uncertain overlapping control systems. In Proc. of the 49th IEEE Conference on Decision and Control, pages 6908-6913, 2010.

[2] A. Ajorlou, A. Momeni, and A. G. Aghdam. A class of bounded distributed control strategies for connectivity preservation in multi-agent systems. IEEE Transactions on Automatic Control, 55(12):2828-2833, 2010.

[3] A. Ajorlou, A. Momeni, and A. G. Aghdam. Sufficient conditions for the convergence of a class of nonlinear distributed consensus algorithms. Automatica, 2011 (to appear).

[4] J. Choi, S. Oh, and R. Horowitz. Distributed learning and cooperative control for multi-agent systems. Automatica 45(12):2802-2814, 2009.

[5] J. Cortes, S. Martinez, and F. Bullo. Robust rendezvous for mobile autonomous agents via proximity graphs in arbitrary dimensions. IEEE Transactions on Automatic Control, 51(8):1289-1298, 2006.

[6] D. V. Dimarogonas, M. Egerstedt, and K. J. Kyriakopoulos. A leader-based containment control strategy for multiple unicycles. In Proc. of the 45th IEEE Conference on Decision and Control, pages 5968-5973, 2006.

[7] J. M. Dion, C. Commault, and J. Woude. Generic properties and control of linear structured systems: a survey. Automatica, 39:1125-1144, 2003.

[8] A. Frank. Rooted k-connections in digraphs. Discrete Applied Mathematics, 157(6):1242-1254, 2009.

[9] F. Gavril. Algorithms for minimum coloring, maximum clique, minimum covering by cliques and maximum independent set of a chordal graph. SIAM J. on Computing, 1:180-187, 1972.

[10] T. Gustavi, D. V. Dimarogonas, M. Egerstedt, and X. Hu. Sufficient conditions for connectivity maintenance and rendezvous in leader-follower networks. Automatica, 46(1):133-139, 2010.

[11] H. Ito, K. Makino, K. Arata, S. Honami, Y. Itatsu, and S. Fujishige. Source location problem with flow requirements in directed networks. Optimization Methods and Software, 18(4):427-435, 2003.
[12] S. Jafari, A. Ajorlou, A. G. Aghdam, and S. Tafazoli. On the structural controllability of multi-agent systems subject to failure: A graph-theoretic approach. In Proc. of the 49 th IEEE Conference on Decision and Control, pages 4565-4570, 2010.

[13] M. Ji and M. Egerstedt. A graph-theoretic characterization of controllability for multi-agent systems. In Proc. of the American Conrol Conference, pages 4588-4593, 2007.

[14] Z. Ji, Z. Wang, H. Lin, and Z. Wang. Interconnection topologies for multi-agent coordination under leader-follower framework. Automatica, 45(12):2857-2863, 2009.

[15] M. Labbe, D. Peeters, and J. F. Thisse. Location on networks. In M.O. Ball et al. (Eds.), Handbooks in OREMS, volume 8, pages 551-624, 1995.

[16] J. Lavaei, A. Momeni, and A. G. Aghdam. A model predictive decentralized control scheme with reduced communication requirement for spacecraft formation. IEEE Transactions on Control Systems Technology, 16(2):268-278, 2008.

[17] Z. Lin, B. Francis, and M. Maggiore. Necessary and sufficient graphical conditions for formation control of unicycles. IEEE Transactions on Automatic Control, 50(1):121-127, 2005.

[18] B. Liu, T. Chu, L. Wang, and G. Xie. Controllability of a leader-follower dynamic network with switching topology. IEEE Transactions on Automatic Control, 53(4):1009-1013, 2008.

[19] B. Liu, G. Xie, T. Chu, and L. Wang. Controllability of interconnected systems via switching networks with a leader. In IEEE international conference on systems, man, and cybernetics, pages 3912-3916, 2006.

[20] X. Liu, H. Lin, and B. M. Chen. A graph-theoretic characterization of structural controllability for multi-agent system with switching topology. In Proc. of the 48th IEEE Conference on Decision and Control, pages 7012-7017, 2009.

[21] S. Martinez, J. Cortes, and F. Bullo. Motion coordination with distributed information. IEEE Control Systems Magazine, 27(4):75-88, 2007.

[22] S. Martini, M. Egerstedt, and A. Bicchi. Controllability analysis of multi-agent systems using relaxed equitable partitions. International Journal of Systems, Control and Communications, 2:100-121, 2010.

[23] M. Mesbahi. On state-dependent dynamic graphs and their controllability properties. IEEE Transactions on Automatic Control, 50(3):387-392, 2005.

[24] N. Moshtagh, N. Michael, A. Jadbabaie, and K. Daniilidis. Vision-based distributed control laws for motion coordination of nonholonomic robots. IEEE Transactions on Robotics, 25(4):851-860, 2009.

[25] H. Nagamochi, T. Ishii, and H. Ito. Minimum cost source location problem with vertex-connectivity requirements in digraphs. Information Processing Letter, 80:287-294, 2001.

[26] R. Olfati-Saber. Flocking for multi-agent dynamic systems: Algorithms and theory. IEEE Transactions on Automatic Control, 51(3):401-420, 2006.

[27] A. Partovi, H. Lin, and J. Zhijian. Structural controllability of high order dynamic multi-agent systems. In IEEE Conference on Robotics Automation and Mechatronics, pages 327-332, 2010 .

[28] A. Rahmani, M. Ji, M. Mesbahi, and M. Egerstedt. Controllability of multi-agent systems from a graph-theoretic perspective. SIAM Journal on Control and Optimization, 48(1):162-186, 2009 
[29] A. Rahmani and M. Mesbahi. On the controlled agreement problem. In Proc. of the American Control Conference, pages 1376-1381, 2006.

[30] J. Shao, G. Xie, and L. Wang. Leader-following formation control of multiple mobile vehicles. IET Control Theory and Applications, 1(2):545-552, 2007.

[31] D. D. Siljak. Decentralized control of complex systems. Academic Press, New York, 1991.

[32] H. Tamura, M. Sengoku, S. Shinoda, and T. Abe. Some covering problems in location theory on flow networks. IEICE Transactions Fundamentals, E75-A(6):678-683, 1992.

[33] H. Tanner. On the controllability of nearest neighbor interconnections. In Proc. of the 43rd IEEE Conference on Decision and Control, pages 2467-2472, 2004.

[34] L. Wang, F. Jiang, G. Xie, and Z. Ji. Controllability of multi-agent systems based on agreement protocols. Science in China Series F: Information Sciences, pages 2074-2088, 2009.

[35] P. Yang, R. A. Freeman, and K. M. Lynch. Multi-agent coordination by decentralized estimation and control. IEEE Transactions on Automatic Control, 53(11):2480-2496, 2008.

[36] M. Zamani and H. Lin. Structural controllability of multiagent systems. In Proc. of the American Control Conference, pages 5743-5748, 2009. 